\title{
Determination of a Depolymerized Holothurian Glycosaminoglycan in Plasma after Intravenous Administration by Postcolumn HPLC
}

\author{
Yong Huang, Hidenao Toyoda, Ichiro KoshiIshi, Toshihiko ToIda, and Toshio Imanari* \\ Faculty of Pharmaceutical Sciences, Chiba University, 1-33, Yayoi-cho, Inage-ku, Chiba 263, Japan. \\ Received July 14, 1995; accepted August 21, 1995
}

\begin{abstract}
Depolymerized holothurian glycosaminoglycan (DHG) produced artificially from sea cucumber has an anticoagulant and antithrombotic activity. In the present study, we attempted to determine the plasma level of DHG using a chemical procedure. A general method for determination of chondroitin sulfates by forming unsaturated disaccharides with chondroitinase digestion was difficult to apply to DHG, because it was resistant to any chondroitinase digestion. We therefore developed a highly sensitive postcolumn HPLC method for determination of intact DHG. DHG was applied to Asahipak $\mathrm{NH}_{2} \mathrm{P}-50$, an amino-bonded column, eluted by alkaline solution and then detected with arginine under a strong alkaline condition as a postcolumn reagent. The limit of detection for DHG was $10 \mathrm{ng}$. The present method was applicable to the determination of DHG in plasma after intravenous administration.
\end{abstract}

Key words antithrombotic agent; sea cucumber; fucose-branched glycosaminoglycan; postcolumn HPLC; arginine; fluorescence detection

Heparin is a very important anticoagulant and antithrombotic agent and has widely been used in the clinical field for over a half century. It does have significant problems, however, such as hemorrhagic complications and being difficult to control for maintaining a therapeutic dosage. To overcome these undesirable effects, a depolymerized heparin (low molecular weight heparin) has recently been developed for clinical use. ${ }^{1,2)}$

Non-heparin glycosaminoglycans (GAGs) have also been studied recently as new anticoagulant and antithrombotic agents. Dermatan sulfate and low molecular weight dermatan sulfate are under clinical trials for new antithrombotic agents in Europe. ${ }^{3,4)}$ The GAG isolated from holothurian (Stichopus Japonicus), being an unique structure of fucose-branched chondroitin sulfates, has an anticoagulant and antithrombotic activity, ${ }^{5-7)}$ but it also induces platelet aggregation. ${ }^{8)}$ In an effort to reduce this property, a depolymerized holothurian glycosaminoglycan (DHG) has been produced by oxidative depolymerization with hydrogen peroxide. ${ }^{9)}$ DHG has anticoagulant and antithrombotic activity without causing platelet aggregation ${ }^{7)}$ and is currently applied for clinical trials as a new antithrombotic agent.

The basic structure of DHG was fucose-branched chondroitin 4,6-sulfates as shown in Fig. 1.9) These fucoses were also sulfated at different positions, which makes the DHG structure heterogeneous. The molecular weight of DHG was about $12 \mathrm{kDa} .{ }^{9)}$ Its anticoagulant actions have recently been elucidated as two different mechanisms, the heparin cofactor II-dependent inhibition of thrombin, and the antithrombin III and heparin cofactor II-independent inhibition of the activation of factor $X$ by the factor IXa-factor VIIIa complex. ${ }^{10)}$

Determination of the plasma level of DHG is very important for study of its pharmacokinetics and for clinical drug monitoring. The current methods of determining heparin or dermatan sulfate in plasma are bioassay and radioactive labeling. Few chemical methods have been reported for analysis of GAG in plasma after administration, because the GAG concentration used was

* To whom correspondence should be addressed. very low and it had to be distinguished from endogenous GAGs. To determine chondroitin sulfates in plasma and urine, fluorometric HPLC methods have already been established after producing unsaturated disaccharides from the GAG by chondroitinase digestion. ${ }^{11,12)}$ However, DHG is resistant to direct chondroitinase digestion. We attempted to produce the unsaturated disaccharides from DHG by chondroitinase after mild acidic hydrolysis to remove fucose as reported by Vieira and Mourão ${ }^{13)}$ and Kariya, et $a l .{ }^{14)}$ This method was applicable to determination of purified DHG, but it was difficult to maintain the reproducibility for plasma samples. Here, we developed a postcolumn HPLC method with arginine as a detection reagent for determination of $\mathrm{DHG}$ concentration in plasma.

\section{Experimental}

Materials DHG (lot No., E920121G) was kindly supplied by Taiho Pharmaceutical Co., Japan. L-Arginine, 2-cyanoacetamide, ethylenediamine monohydrate, taurine, benzamidine hydrochloride hydrate and taurocyamine were purchased from Wako Pure Chemical Industry, Japan; Actinase was from Kaken Pharmaceutical Co., Tokyo, Japan. All other reagents were of analytical grade.

Apparatus and HPLC Conditions A flow diagram of liquid chromatography for determination of DHG is shown in Fig. 2. The system was assembled with gradient pumps (Jusco 880-PU, intelligent HPLC pump), a sample injector (VMD-350, Shimamura Instrument Co.), a double-plunger pump (PSU-2.5W, Seishin Pharmaceutical, Tokyo, Japan) for the reagent solution, a dry reaction bath (Type DB-3,

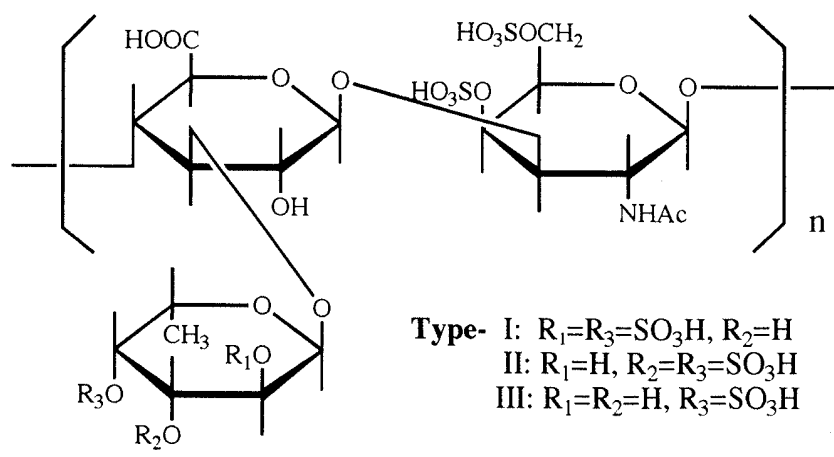

Fig. 1. Structure of DHG

(C) 1995 Pharmaceutical Society of Japan 


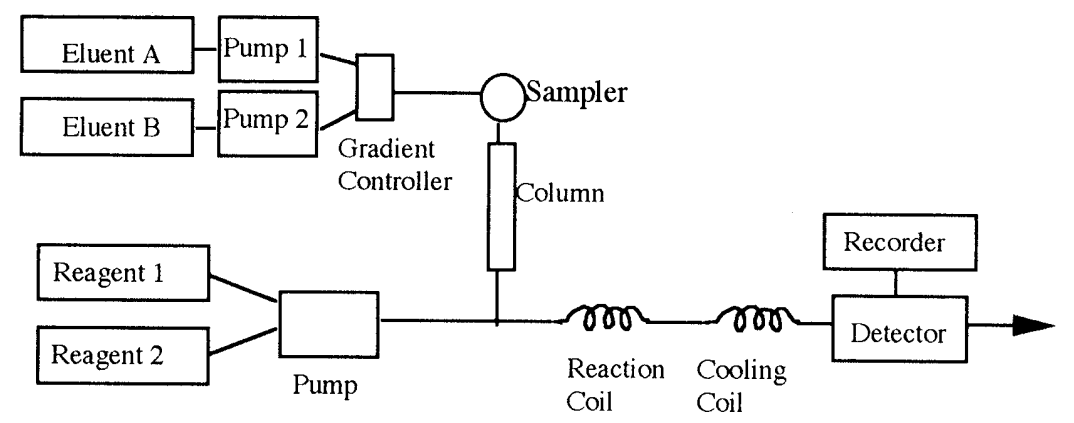

Fig. 2. Flow Diagram of the Postcolumn HPLC System for Determination of DHG

Column, Asahipak $\mathrm{NH}_{2} \mathrm{P}-50(4.6 \mathrm{~mm}$ i.d. $\times 250 \mathrm{~mm})$ at room temperature; eluent A, $0.1 \mathrm{M}$ phosphate buffer $(\mathrm{pH} 10.0)$ containing $0.1 \mathrm{M} \mathrm{NaCl}$; eluent B, $0.1 \mathrm{M}$ phosphate buffer ( $\mathrm{pH} 10.0$ ) containing $0.5 \mathrm{M} \mathrm{NaCl}$; gradient, $0-10 \mathrm{~min}, 0-100 \% \mathrm{~B} ; 10-20 \mathrm{~min}, 100 \% \mathrm{~B} ; 20-25 \mathrm{~min}, 100-0 \% \mathrm{~B}$; flow rate, $0.5 \mathrm{ml} / \mathrm{min} ; \mathrm{reagent} 1,0.5 \mathrm{M} \mathrm{NaOH}$ $(0.25 \mathrm{ml} / \mathrm{min})$; reagent $2,1 \%$ arginine $(0.25 \mathrm{ml} / \mathrm{min})$; reaction temperature, $110^{\circ} \mathrm{C}$; reaction coil, $0.5 \mathrm{~mm}$ i.d. $\times 10 \mathrm{~m}$; cooling coil, $0.25 \mathrm{~mm}$ i.d. $\times 2 \mathrm{~m}$; detection, Ex. $325 \mathrm{~nm}$, Em. $420 \mathrm{~nm}$.

Shimamura Instrument Co.), fluorescence spectrophotometer (Hitachi F-1050) and a chromatointegrator (Hitachi, D-2500). Chromatographic column, Asahipak $\mathrm{NH}_{2} \mathrm{P}-50,(250 \mathrm{~mm} \times 4.6 \mathrm{~mm})$ was obtained from Shodex Co., Japan. Mobile phase consisted of eluent A, $0.1 \mathrm{M}$ phosphate buffer $(\mathrm{pH} 10.0)$ containing $0.1 \mathrm{M} \mathrm{NaCl}$ and eluent $\mathrm{B}, 0.1 \mathrm{M}$ phosphate buffer ( $\mathrm{pH} 10.0$ ) containing $0.5 \mathrm{M} \mathrm{NaCl}$. A linear gradient program was started at eluent B from $0 \%$ to $100 \%$ within $10 \mathrm{~min}$, and kept at $100 \%$ until $20 \mathrm{~min}$, then reduced to $0 \%$ at $25 \mathrm{~min}$. Flow rate was consistent at $0.5 \mathrm{ml} / \mathrm{min}$. To the eluate were added $0.5 \mathrm{M} \mathrm{NaOH}$ and $1 \%$ arginine solution at a flow rate of $0.25 \mathrm{ml} / \mathrm{min}$ using a double-plunger pump. The mixture passed through a polytetrafluoroethylene (PTFE) reaction coil $(0.5 \mathrm{~mm}$ i.d. $\times 10 \mathrm{~m})$ set in a dry reaction bath thermostated at $110^{\circ} \mathrm{C}$ and then a PTFE cooling coil $(0.25 \mathrm{~mm}$ i.d. $\times 2 \mathrm{~m})$. The effluent was monitored by the fluoromonitor with excitation, $325 \mathrm{~nm}$ and emission, $420 \mathrm{~nm}$.

Measurement of Fluorescence Spectra of the Reactants of DHG Fluorescence spectra of the reaction products formed by DHG and fluorescence reagent were obtained as follows: To a sealed test tube, $2 \mathrm{ml}$ of DHG solution $(25 \mu \mathrm{g} / \mathrm{ml}), 1 \mathrm{ml}$ of fluorescence reagent and $1 \mathrm{ml}$ of $0.5 \mathrm{M} \mathrm{NaOH}$ was added and heated at $100^{\circ} \mathrm{C}$ for $5 \mathrm{~min}$. Immediately after cooling, fluorescence spectra were measured by a fluorescence spectrophotometer (Hitachi, MPF-4), with scanning speed, $60 \mathrm{~nm} / \mathrm{min}$; chart speed, $30 \mathrm{~mm} / \mathrm{min}$.

Animal Experiments Male albino rabbits weighing $3.5 \pm 0.6$ (mean \pm S.D.) $\mathrm{kg}$ were used for the study. DHG saline solution $(2 \mathrm{mg} / \mathrm{ml})$ was given intravenously $(1 \mathrm{mg} / \mathrm{kg})$ by a bolus injection through the marginal ear vein. Blood samples $(300-400 \mu l)$ were taken from the marginal ear vein on the other side at $0,5,15,30,45,60,120 \mathrm{~min}$ after administration. Plasma was obtained by centrifugation with $2.5 \%$ EDTA as an anticoagulant agent.

Extraction of DHG from Plasma DHG in plasma was separated by our modified method reported previously. ${ }^{15)}$ To a $100 \mu \mathrm{l}$ portion of plasma sample, $100 \mu \mathrm{l}$ of $0.05 \mathrm{M}$ Tris- $\mathrm{HCl}$ buffer $(\mathrm{pH} 8.0)$ containing $1 \%$ actinase was added and the mixture was digested at $45^{\circ} \mathrm{C}$ for $3 \mathrm{~h}$. One and a half $\mathrm{ml}$ of $0.015 \mathrm{M}$ acetic acid containing $10 \% \mathrm{NaCl}$ was then added and the mixture was heated in a boiling water bath for $5 \mathrm{~min}$. After cooling, the solution was centrifuged at $2300 \times \mathbf{g}$ for $15 \mathrm{~min}$. The supernatant was transferred to another tube and $5 \mathrm{ml}$ of cooled ethanol saturated with sodium acetate was added. The solution was kept at $0^{\circ} \mathrm{C}$ for $1 \mathrm{~h}$ and centrifuged at $2300 \times g$ for $15 \mathrm{~min}$ at $4^{\circ} \mathrm{C}$. The precipitate was lyophilized and then dissolved in $40 \mu \mathrm{l}$ of distilled water. An aliquot $(10-20 \mu 1)$ of the sample was submitted to HPLC.

\section{Results}

Detection of DHG by Postcolumn HPLC To establish a highly sensitive detection system for DHG, the available postcolumn fluorescence reagents, $2 \%$ arginine, ${ }^{16)} 20 \mathrm{~mm}$ taurocyamine, ${ }^{17)} 1 \%$ 2-cyanoacetamide, ${ }^{18)} 15 \mathrm{~mm}$ ethylenediamine, ${ }^{19)} 100 \mathrm{~mm}$ benzamidine ${ }^{20)}$ and $8 \%$ taurine $^{21)}$ were examined. DHG reacted with arginine, taurocyamine and 2-cyanoacetamide giving a strong fluorescence, but the fluorescence intensity of arginine was about 5 and 1.3

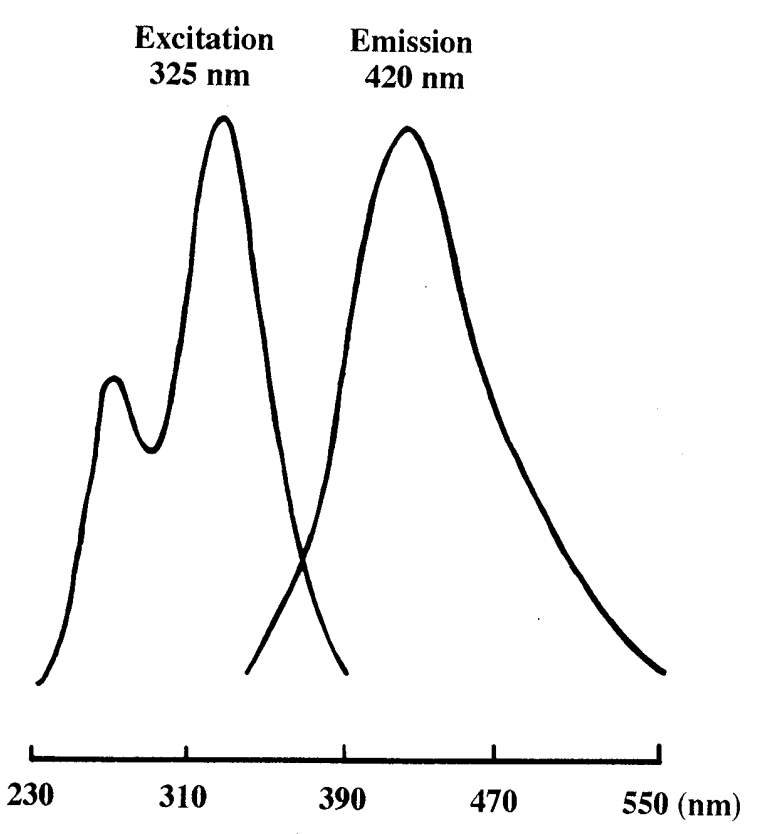

Fig. 3. Fluorescence Spectra of the Product Obtained from the Reaction of DHG with Arginine under Strong Alkaline Conditions at $100^{\circ} \mathrm{C}$

times higher than that of 2-cyanoacetamide and taurocyamine respectively. Therefore, arginine was thought to be a better fluorescence reagent for DHG detection. Figure 3 shows the excitation and fluorescence spectra of the product of DHG heated with arginine under a strong alkaline condition. Very low fluorescence intensity was observed when $2.5 \%$ boric acid was used ${ }^{16)}$ instead of $\mathrm{NaOH}$ solution for the reaction of $\mathrm{DHG}$ and arginine. This experiment indicated that an alkaline condition was very important for the fluorescence reaction of DHG.

The postcolumn HPLC conditions were established as shown in Fig. 2. The optimum reaction temperature was $110^{\circ} \mathrm{C}$ (Fig. 4). The optimum concentrations of arginine and $\mathrm{NaOH}$ were further examined and determined to be $1 \%$ and $0.5 \mathrm{M}$, respectively (data not shown).

Separation of DHG by HPLC Since a considerable amount of low-sulfated chondroitin 4-sulfate (LSC) is present in plasma (its concentration in human plasma is $13 \mu \mathrm{g} / \mathrm{ml}),{ }^{15}$ DHG must be separated from LSC to determine its quantity in plasma. As a high degree of sulfation in DHG structure, it can be separated DHG 
from LSC and other interfering substances by ionexchange chromatography. A strong anion exchange HPLC column has been used to separate GAG. ${ }^{2,23)}$ However, DHG was adsorbed to the column so strongly that an eluate with high ionic strength $(\mathrm{NaCl}>2.0 \mathrm{M})$ had to be used. Asahipak $\mathrm{NH}_{2} \mathrm{P}-50$ column, an amino-bonded vinyl alcohol copolymer gel, is a weak anion exchange column and stable in alkaline solvent $(\mathrm{pH}$ range of the mobile phase was allowed from 2 to 13). The retention capacity of the column was dependent on the degree of ionized amine group, which was easily controlled by the $\mathrm{pH}$ of eluting solvent. Therefore, it was possible to elute DHG from this column even with a relatively low concentration of $\mathrm{NaCl}$ in alkaline buffer. DHG could be resolved well from LSC using the mobile phase of $0.1 \mathrm{M}$ phosphate buffer ( $\mathrm{pH} 10.0$ ) containing $0.1-0.5 \mathrm{M} \mathrm{NaCl}$ with linear gradient elution (Fig. 5). The retention time of DHG was $18.0 \mathrm{~min}$. Under this condition, DHG could also be separated from chondroitin sulfates, dermatan sulfates and heparan sulfates, which showed the same retention times (14 min). The limit of detection for DHG was $10 \mathrm{ng}(S / N=3)$. A good linear relationship was obtained between the peak height and DHG amount from

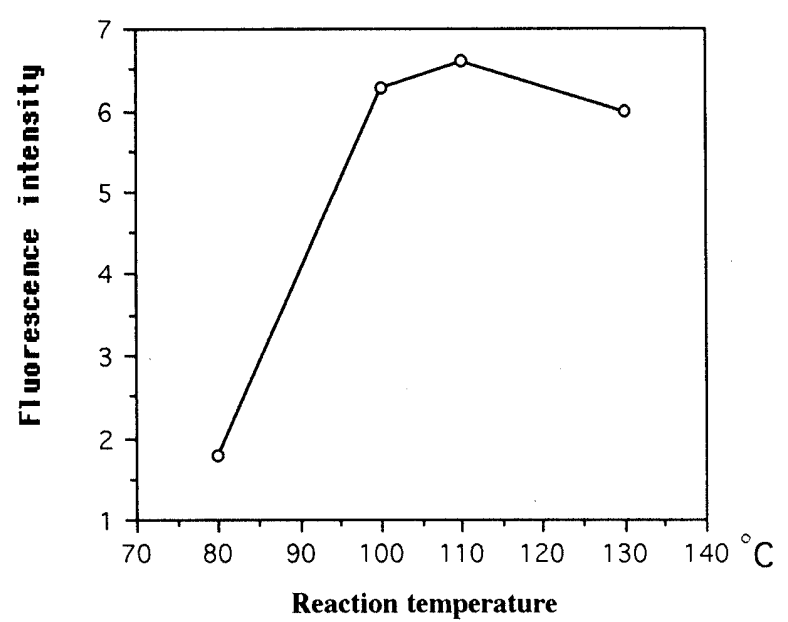

Fig. 4. Effect of Temperature on the Reaction of DHG with Arginine Other conditions are described in the text.
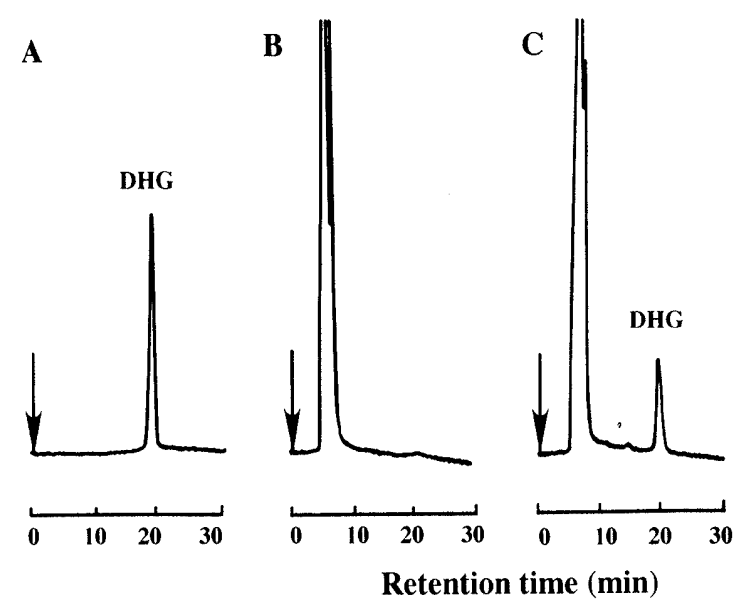

$100 \mathrm{ng}$ to $10 \mu \mathrm{g}$ (the regression coefficient was 1.0 ). The peak height and retention time of DHG varied less than $3 \%$ after repeated injections of the same amount at $500 \mathrm{ng}$ $(n=6)$.

Extraction of DHG from Plasma Various amounts of DHG were spiked to $100 \mu$ plasma samples and DHG was separated by the method described above. Briefly, DHG was extracted with acidic solution containing $\mathrm{NaCl}$ after boiling plasma samples to eliminate proteins. The GAGs in the supernatant were separated and then precipitated with ethanol. The decomposition of plasma proteins with actinase was a necessary step for DHG extraction. The extraction recovery of DHG with this treatment was about $70 \%$ (see Table 1). The chromatogram of DHG in plasma is shown in Fig. 5. DHG could be separated from LSC and plasma proteins completely. The calibration curve of DHG in plasma is shown in Fig. 6. There was a good linear relationship between the peak height and plasma DHG concentration from $0.5 \mu \mathrm{g} / \mathrm{ml}$ to $100 \mu \mathrm{g} / \mathrm{ml}$ ( $100 \mu \mathrm{l}$ plasma was used for analysis). The reproducibility of DHG determination for plasma sample is shown in Table 1. The coefficients of variation (C.V.) values are less than $6 \%(n=5)$.

Pharmacokinetics of DHG after Intravenous Administration The time course experiment of DHG level in rabbit plasma after intravenous administration of $1 \mathrm{mg} / \mathrm{kg}$ is shown in Fig. 7. The $t_{1 / 2}$ of DHG was $15.5 \pm 2.8$ $(n=4) \mathrm{min}$, which indicated DHG was rapidly eliminated from plasma. From the chromatogram, we could not observe any metabolic peak caused by oligosaccharides of DHG.

\section{Discussion}

HPLC analysis of intact GAGs with precolumn labeling derivation $^{23)}$ and capillary electrophoresis analysis of intact GAGs have recently been reported. ${ }^{24)}$ In this work, we developed a highly sensitive postcolumn HPLC method for determination of DHG in plasma.

Several postcolumn fluorescence reagents were used to obtain this highly sensitive method. Arginine was found suitable for DHG determination, but the reaction mechanism of DHG with arginine is still uncertain. The

Fig. 5. Chromatograms of Standard DHG (A); Blank Plasma (B); Blank Plasma Spiked DHG (C); Plasma Sample Taken 30 min after i.v. Administration of $1 \mathrm{mg} / \mathrm{kg}$ DHG in Rabbit (D)

HPLC conditions are described in the text. 
Table 1. Extraction Recoveries and Reproducibilities of DHG in Plasma

\begin{tabular}{lcc}
\hline \hline DHG concentration & 5 & 50 \\
& & $(\mu \mathrm{g} / \mathrm{ml})$ \\
\hline Peak height \pm S.D. & $2.86 \pm 0.12$ & $29.5 \pm 1.6$ \\
C.V. $(n=5)$ & $4.20 \%$ & $5.30 \%$ \\
Extraction recoveries $^{a)}$ & $72.20 \%$ & $74.50 \%$
\end{tabular}

a) Extraction recoveries were calculated by comparing the peak height of DHG extracted from plasma with the peak height of the same amount of standard DHG. DHG in plasma was extracted as described in the text.

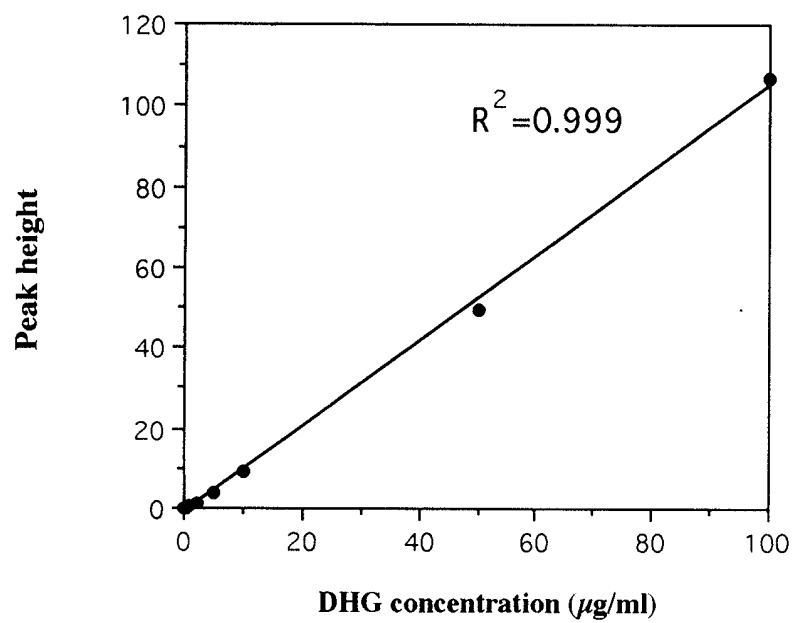

Fig. 6. Relationship between DHG Concentration in Plasma and Peak Height

DHG was extracted according to the method described in the text. Plasma concentration of DHG was $0.5-100 \mu \mathrm{g} / \mathrm{ml}$

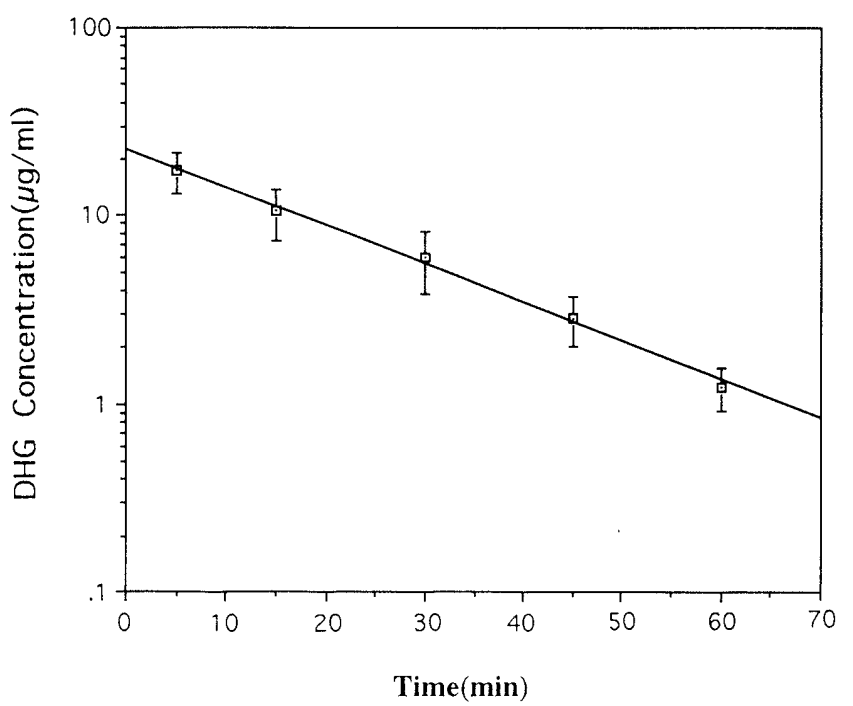

Fig. 7. Plasma Concentration of DHG in Rabbits after i.v. Administration of DHG, $1 \mathrm{mg} / \mathrm{kg}$

Data are mean \pm S.E. of 4 rabbits.

reaction condition reported here differed from that reported by Mikami and Ishida, ${ }^{16)}$ in which arginine reacted with reducing sugars in boric acid solution at neutral $\mathrm{pH}$. Richards and Sephton ${ }^{25}$ reported that polysaccharides could be degraded by boiling in $0.5 \mathrm{M}$ $\mathrm{NaOH}$ solution; therefore, it is very possible that DHG would be degraded under strong alkaline conditions. The degradation products may react with arginine to form fluorescence derivatives.

DHG has such strong negative charges that it would bind to some proteins in plasma as heparin does. ${ }^{26)}$ This property makes its extraction from plasma difficult. We examined the pretreatment methods reported by Oreste and Stella ${ }^{27)}$ and Hata et al. ${ }^{28)}$ and found that the method described here achieved the highest extraction recovery of DHG. Endogenous GAGs in plasma (mainly LSC) had also been simultaneously extracted with DHG by this pretreatment, but they could be well resolved in ion-exchange chromatography as shown in Fig. 5. The Asahipak $\mathrm{NH}_{2} \mathrm{P}-50$ column was recently developed as a new kind of amino column which is especially useful for separation of carbohydrates. Here, we used a weak alkaline mobile phase for DHG analysis which has the following advantages: 1) DHG could be eluted faster with a relatively low concentration of $\mathrm{NaCl}$ because the positive charge of the column was reduced in the alkaline phase; 2) The mobile phase contained a low concentration of $\mathrm{NaCl}$ which would increase the reaction rate of $\mathrm{DHG}$ with arginine and fluorescence intensity; 3) The higher theoretical plates of this column were obtained in alkaline mobile phases at $\mathrm{pH} 10.0$.

In conclusion, this method is highly specific and sensitive enough for DHG determination in plasma. The advantage is that the plasma proteins and endogenous GAG do not interfere with DHG quantitation. It can be used not only for determination of DHG in plasma but also for in other biological samples such as urine, and may also be used for DHG quality control during manufacturing.

\section{References}

1) Ehrlich J., Stivala S. S., J. Pharm. Sci., 62, 517 (1973)

2) Green D., Hirsh J., Heit J., Prins M., Davidson B., Lensing A. W. A., Pharmacol. Rev., 46, 89 (1994).

3) Linhardt R. J., Al-Hakim A., Liu J., Hoppensteadt D., Mascellani G., Bianchini P., Fareed J., Biochem. Pharmacol., 42, 1609 (1991).

4) Linhardt R. J., Desai U. R., Liu J., Pervin A., Hoppensteadt D., Fareed J., Biochem. Pharmacol., 47, 1241 (1994).

5) Li J., Bao C., Chen G., Zhang G., Zhang J., Fan H., Chen J., Hao X., Zhongyao Tongbao (Chinese), 8, 36 (1983).

6) Li J., Bao C., Chen G., Zhang G., Fan H., Chen J., Hao X., Zhongguo Yaoli Xuebao (Chinese), 6, 107 (1985).

7) Suzuki N., Kitazato K., Takamatsu J., Saito H., Thromb. Haemostas., 65, 369 (1991).

8) Li J., Lian E. C. Y., Thromb. Haemostas., 59, 435 (1988).

9) Yoshida K., Minami Y., Nemoto H., Numata K., Yamanaka E., Tetrahedron Lett., 33, 4959 (1992).

10) Nagase H., Enjyoji K., Minamiguchi K., Kitazato K. T., Kitazato K., Saito H., Kato H., Blood, 85, 1527 (1995).

11) Toyoda H., Shinomiya K., Yamanashi S., Koshiishi I., Imanari T., Anal. Sci., 4, 381 (1988).

12) Toyoda H., Motoki K., Tanikawa M., Shinomiya K., Akiyama H., Imanari T., J. Chromatogr., 565, 141 (1991).

13) Vieira R. P., Mourão P. A. S., J. Biol. Chem., 263, 18176 (1988).

14) Kariya Y., Watabe S., Hashimoto K., Yoshida K., J. Biol. Chem., 265, 5081 (1990).

15) Huang Y., Toyoda H., Toida T., Imanari T., Biomed. Chromatogr., 9, 102 (1995).

16) Mikami H., Ishida Y., Bunseki Kagaku, 32, E207 (1983).

17) Kinoshita T., Kamitani Y., Yoshida J., Urano T., Nimura N., Hanai T., J. Liquid Chromatogr., 14, 1929 (1991).

18) Honda S., Matsuda Y., Takahashi M., Kakehi K., Ganno S., Anal. Chem., 106, 238 (1980).

19) Honda S., Kakimoto K., Sudo K., Kakehi K., Takiura K., Anal. Chim. Acta., 70, 133 (1983)

20) Kai M., Tamura K., Yamaguchi M., Ohkura Y., Anal. Sci., 1, 59 
(1985).

21) Kato T., Kinoshita T., Chem. Pharm. Bull., 26, 1291 (1978).

22) Rice K. G., Linhardt R. J., Carbohydr. Res., 190, 219 (1989).

23) Takagaki K., Takeda Y., Nakamura T., Daidouji K., Narita H., Endo M., J. Biochem. Biophys. Methods, 28, 313 (1994).

24) Honda S., Ueno T., Yasueda S., Nishiura S., Tachibana S., Eleventh Symposium on Analytical Chemistry of Biological Substances of
Japan, Funabashi, 1994, p. 47.

25) Richards G. N., Sephton H. H., J. Chem. Soc., 1957, 4492.

26) Becker K. E., Gott V. L., Allen J. C., Amer. J. Physiol., 218, 1500 (1970).

27) Oreste P. M., Stella P., Anal. Biochem., 210, 136 (1993).

28) Hata R. I., Ohkawa S. I., Nagai Y., Biochim. Biophys. Acta, 543, 156 (1978). 